\title{
Effects of Different Post-Activation Potentiation Warm-Ups on Repeated Sprint Ability in Soccer Players from Different Competitive Levels
}

\author{
by \\ Javier Sanchez-Sanchez ${ }^{1}$, Alejandro Rodriguez2, Cristina Petisco ${ }^{1}$, \\ Rodrigo Ramirez-Campillo ${ }^{3,4}$, Cristian Martínez ${ }^{5}$, Fábio Y. Nakamura ${ }^{6,7}$
}

This study aimed to compare the effects of a traditional warm-up with two post-activation potentiation (PAP) warm-up strategies on the repeated sprint ability (RSA) of soccer players from national (NL) and regional (RL) competitive levels. Sixteen young players (NL, $n=8$, age $=20.7 \pm 1.4 \mathrm{y}$, body mass $=68.5 \pm 7.0 \mathrm{~kg}$, body height $=177.4$ $\pm 5.2 \mathrm{~cm} ; R L, n=8$, age $=20.8 \pm 1.0 \mathrm{y}$, body mass $=68.7 \pm 4.0 \mathrm{~kg}$, body height $=176.6 \pm 5.6 \mathrm{~cm})$ were recruited to complete a traditional warm-up (CONTROL), a PAP warm-up incorporating squats with a load ( 60\% 1RM) that allowed a high speed $(1 \mathrm{~m} / \mathrm{s})$ of movement and a high number of repetitions (PAP-1), and a PAP warm-up with a load $(\sim 90 \% 1 R M)$ that allowed a moderate speed $(0.5 \mathrm{~m} / \mathrm{s})$ of movement and a reduced number of repetitions (PAP-0.5). A RSA test (six 20-m sprints with $20 \mathrm{~s}$ of recovery) was performed 5 min after the PAP warm-up to assess the effects of the different protocols on the fastest sprint $(R S A b)$ and the mean time of all sprints (RSAm). A meaningful improvement of RSA performance was observed with PAP-0.5, attaining a large effect on $N L$ ( $R S A b, E S=-1.5 ; R S A m$, $E S=-1.3)$ and only a small effect on RL athletes (RSAb and RSAm, ES =-0.2). Moreover, when each RSA sprint performance was compared between NL and RL players, after PAP-0.5 greater performance for all sprints was observed in the NL players. Therefore, adding a heavy strength-based conditioning exercise during the warm-up prior to a RSA test may induce significant performance improvements in NL, but only small effects in RL players.

Key words: soccer, explosive actions, fatigue, back squats, athletes.

\section{Introduction}

The ability to sustain repeated explosive actions during a match is a key issue in soccer (Stolen et al., 2005; Turner et al., 2015). Improvement of these explosive performances (e.g., sprinting speed) have been chronically reported after muscular strength training programs (Hoff and Helgerud, 2004), although acute improvements may also be obtained under specific conditions (Wilson et al., 2013). Apart from reducing the injury risk (Woods et al., 2007), a warm-up can also acutely improve explosive performance. However, more traditional warmup strategies usually encompass low-intensity endurance exercise (e.g., running), flexibility and sport-specific drills (Schilling and Stone, 2000), and may not optimize explosive strength (Chiu et

\footnotetext{
1 - Pontifical University of Salamanca, Salamanca, Spain.

2 - Isabel I Universtiy, Burgos, Spain.

3 - Department of Physical Activity Sciences, Universidad de Los Lagos, Osorno, Chile.

4 - Nucleus of Health, Physical Activity and Sports, Laboratory of Measurement and Assessment of Sports Performance, Universidad de Los Lagos, Osorno, Chile.

5 - Department of Physical Education, Sport and Recreation, University of La Frontera, Temuco, Chile.

6 - Department of Medicine and Aging Sciences, "G. d'Annunzio" University of Chieti-Pescara, Chieti, Italy.

7 - The College of Healthcare Sciences, James Cook University, Queensland, Australia.
} 
al., 2003). In soccer, warm-up strategies should aim to increase muscle temperature (Bishop, 2003) and neural activation (Zois et al., 2015), while minimizing fatigue. In this sense, a warm-up incorporating post-activation potentiation (PAP) may be an effective strategy compared to a traditional warm-up (Gouvea et al., 2013; Zois et al., 2011, 2015) in this sport discipline.

The PAP effect depends on the balance between fatigue and neuromuscular potentiation (Tillin and Bishop, 2009), which in turn depends on the type of exercise, volume, intensity, and recovery time (Baudry and Duchateau, 2007; Masamoto et al., 2003; Robbins, 2005; Sale, 2004). However, the PAP effect may be observed after the application of maximal loaded (Kilduff et al., 2008), submaximal loaded (Wilson et al., 2013) and unloaded exercises (Chaouachi et al., 2010a), especially when performed with the intention of maximizing movement velocity, leading to the recruitment of fast-twitch muscle fiber (Cronin et al., 2002), which is considered a key factor to induce PAP (Turner et al., 2015). However, although both high-loaded low-external speed (Pareja-Blanco et al., 2014) and low-loaded highexternal speed exercises (Turner et al., 2015) may induce PAP, the optimal load is not clear (Tillin and Bishop, 2009). It is important to note that athletes' characteristics may affect the PAP occurrence (Wilson et al., 2013), with higher effects in elite (Chiu et al., 2003), highly trained (Wilson et al., 2013) and stronger athletes (Kilduff et al., 2008).

The type of applied tests to assess the PAP effects should also be considered. Most researchers have used maximal-intensity singleeffort tests (e.g., sprint) (Chatzopoulos et al., 2007; Turner et al., 2015), but scarce information exists regarding PAP effects on the ability to sustain repeated explosive actions, such as during the repeated sprint ability (RSA) test (Okuno et al., 2013; Zois et al., 2015), a key fitness specific-trait for soccer (Spencer et al., 2005), which determines match physical performance (Rampinini et al., 2007) and differentiates between competitive levels (Rampinini et al., 2009).

Therefore, this study aimed to compare the acute effects of a traditional warm-up with two PAP warm-up strategies on the RSA of soccer players from national and regional competitive levels. The study hypothesis was that the two
PAP warm-up strategies would induce greater effects on the RSA of soccer players compared to the traditional warm-up, with greater effects in national-level athletes.

\section{Methods}

\section{Participants}

National level (NL, $\mathrm{n}=8$ ) and regional level ( $R L, n=8$ ) soccer players with $\geq 6$ years of training and competition experience volunteered to participate in this study. Athletes were free of injuries in the last 3 months and had regularly trained and competed in their clubs in the past 6 months. Athletes signed an informed consent form after receiving verbal and written briefing of potential risks and benefits derived from study participation. The study was conducted following the Declaration of Helsinki and approved by the institutional committee from the department responsible for the study (Pontifical University of Salamanca). The descriptive characteristics of participants are shown in Table 1.

\section{Design and Procedures}

The experiment was conducted during the last month of the competitive period. Aside from a familiarization session, athletes completed (with counterbalanced order across the athletes) 3 different warm-up protocols, separated by 48 hours of rest (Taylor et al., 2013). All protocols were performed at the same time of day. Participants were asked to attend each session under an adequate feeding and hydration state. During the whole duration of the experiment, participants did not take part in any training program other than the one applied in their soccer club.

Familiarization and maximal progressive explosive squat test

During the familiarization session, athletes simulated the warm-up protocols and completed a maximal progressive explosive squat test (Gonzalez-Badillo et al., 2015) in order to assess the specific loads to be used during PAP warm-up sessions. Athletes completed a progressive squat test on a Smith machine (MultipowerPeroga ${ }^{\circledR}$, Murcia, Spain), with the barbell constrained to move along the vertical axis. In the initial position the barbell was at shoulder-level, feet at shoulder-width distance, and knee and hips in full extension. Adhesive marks were added to the floor and the barbell to 
assure consistency in the hands and feet position during testing. From the initial position, athletes performed an eccentric movement using a $90^{\circ}$ knee angle (goniometer-determined) with a controlled speed $(0.5-0.65 \mathrm{~m} / \mathrm{s})$ (Pareja-Blanco et al., 2014) and then a concentric leg extension as fast as possible (without jumping). The initial load was $20 \mathrm{~kg}$ (to allow velocities $\geq 1 \mathrm{~m} / \mathrm{s}$ ), for one set of three consecutive repetitions. After finishing the first set, the load was increased by $10 \mathrm{~kg}$ in the following bouts (Gonzalez-Badillo et al., 2015; Sanchez-Medina et al., 2010). Four minutes of rest were allowed between sets. The test ended when subjects attained a given weight that precluded the attainment of a mean propulsive velocity $\geq 0.5$ $\mathrm{m} / \mathrm{s}$ in the three repetitions for a given set (i.e., $\sim 90 \%$ of one repetition maximum [1RM]) (Loturco et al., 2016; Pareja-Blanco et al., 2014). A linear encoder (SmartCoachPowerEncoder ${ }^{\circledR}$, Europe AB. Stockholm, Sweden) and software SmartCoach ${ }^{\circledR}$ (v.3.1.3.09) were used to measure speed during testing and to provide feedback to athletes after each set.

\section{Warm-up protocols}

One of the warm-up protocols was designed (according to athletes regular practice) as the control condition (CONTROL), with 10 minutes of running at $60 \%$ of the maximal heart rate (Zois et al., 2015) and 5 minutes of dynamic stretching (Faigenbaum et al., 2005). The two experimental PAP warm-ups included the same exercises as the CONTROL, with the addition (one minute after the end of the stretching exercises) of one set of explosive squats, performed with the technique previously described. In one of the experimental PAP warmups the squats were performed with a load $(\sim 60 \%$ $1 \mathrm{RM})$ that allowed a high speed $(1 \mathrm{~m} / \mathrm{s})$ of movement (PAP-1) (Gonzalez-Badillo et al., 2015) and the other with a load ( 90\% 1RM) (Kilduff et al., 2008) that allowed a moderate speed $(0.5 \mathrm{~m} / \mathrm{s})$ of movement (PAP-0.5). In both PAP-1 and PAP0.5 athletes completed repetitions until mean propulsive concentric velocity was reduced by $\geq 10 \%$ for each corresponding speed (SanchezMedina and Gonzalez-Badillo, 2011). After the warm-up, athletes rested passively for 5 minutes and then performed the RSA test (Okuno et al., 2013).

RSA test

The RSA test included 6 sprints of $20 \mathrm{~m}$, with a passive recovery period of $20 \mathrm{~s}$ in between (Aziz et al., 2007). Times were recorded with a double beam photocell system (Witty, Microgate ${ }^{\circledR}$, Italy). Athletes received verbal motivation during the test and, $6 \mathrm{~s}$ before each sprint, they were verbally and visually informed to assume the starting position $0.5 \mathrm{~m}$ behind the starting line (Chaouachi et al., 2010b), and from there a countdown of $3 \mathrm{~s}$ was visually provided to athletes with a light panel (Microgate ${ }^{\circledR}$, Italy). The fastest sprint (RSAb) and the mean time of all sprints (RSAm) were retained for further analyses. Statistical analysis

All values are reported as means \pm standard deviations. Normality and homoscedasticity assumptions made for all data were checked using the Shapiro-Wilk and Levene tests, respectively. A two-way analysis of variance (protocol [3 levels], group [2 levels]) was used to compare the effects of warm-up conditions, followed by a Tukey post hoc test. To compare each RSA sprint performance according to the competitive level a two-way analysis (group [2 levels], sprint [6 levels]) was used. Alpha level was set at $p<0.05$. Cohen's d effect size (ES) analysis also was performed, using the following classification system: $\leq 0.19$ (trivial); 0.2-0.59 (small); 0.6-1.19 (moderate); 1.2-1.99 (large); $\geq 2$ (very large) (Hopkins et al., 2009). Analyses were performed with the Statistical Package for the Social Sciences (SPSS v. 21.0; SPSS Inc., USA).

\section{Results}

Maximal progressive explosive squat test

The weight lifted during both PAP-0.5 and PAP-1 and the number of repetitions completed during PAP- 0.5 were greater for the NL compared to the RL athletes (Table 2).

Warm-up effects

No differences were observed between CONTROL and PAP- 0.5 or PAP- 1 for RSAb and RSAm (Table 3). However, compared to CONTROL, a small effect was observed for the reduction in RSAb after PAP-0.5 (ES = -0.4).

Soccer player's competitive level

Although no differences were observed between NL and RL players in RSAb and RSAm in the CONTROL condition, the NL players showed greater RSAb and RSAm performance after PAP-0.5 $(p<0.01)$ and PAP-1 $(p<0.05)$ (Table 3). 
When each RSA test bout was compared between NL and RL players, greater performance was observed in the former under CONTROL condition during sprints $4(\mathrm{ES}=1.4$; Dif $=5.5 \%)$ and $6(\mathrm{ES}=1.2 ;$ Dif $=6.9 \%)(p<0.05)($ Figure 1, A). However, after PAP-0.5 greater performance for all sprints was observed in NL compared to RL players $(p<0.01$ in the sprint 2 [ES $=1.6$; Dif $=$ $5.5 \%], 5[\mathrm{ES}=1.5 ;$ Dif $=6.3 \%]$ and $6[\mathrm{ES}=1.4 ;$ Dif $=$ $6.5 \%] ; p<0.05$ in the sprint 1 [ES $=2.0 ;$ Dif $=3.7 \%$, $3[\mathrm{ES}=2.1 ; \mathrm{Dif}=6.0 \%]$ and $4[\mathrm{ES}=1.7 ; \mathrm{Dif}=6.3 \%]$ sprint) (Figure 1, B). No differences between groups were observed after PAP-1 (Figure 1, C).

Table 1

Characteristics of national $(N L)$ and regional $(R L)$ level soccer players

\begin{tabular}{ccccc}
\hline & $\begin{array}{c}\text { Age } \\
(\mathrm{y})\end{array}$ & $\begin{array}{c}\text { Body } \\
\text { mass } \\
(\mathrm{kg})\end{array}$ & $\begin{array}{c}\text { Body } \\
\text { height } \\
(\mathrm{cm})\end{array}$ & $\begin{array}{c}\text { Soccer-weekly training volume } \\
\text { (hours) }\end{array}$ \\
\hline \multirow{2}{*}{$\mathrm{NL}$} & $\begin{array}{c}20.7 \pm \\
1.4\end{array}$ & $68.5 \pm 7.0$ & $177.4 \pm 5.2$ & $6-8$ \\
$\mathrm{RL}$ & $\begin{array}{c}20.8 \pm \\
1.0\end{array}$ & $68.7 \pm 4.0$ & $176.6 \pm 5.6$ & $3-5$ \\
\hline
\end{tabular}

Table 2

Weight lifted and repetitions completed by national and regional level soccer players during different post-activation potentiation (PAP) warm-ups

\begin{tabular}{|c|c|c|c|c|}
\hline & National level & Regional level & Effect size & $\begin{array}{c}\text { Difference }^{a} \text { between } \\
\text { groups }(\%)\end{array}$ \\
\hline \multicolumn{5}{|l|}{ PAP-0.5 } \\
\hline Weight lifted (kg) & $112.6 \pm 14.3^{\mathrm{b}, \mathrm{c}}$ & $96.5 \pm 10.2^{\mathrm{c}}$ & 1.3 & 14.2 \\
\hline Repetitions & $4.4 \pm 0.5^{\mathrm{b}, \mathrm{c}}$ & $2.9 \pm 0.6^{c}$ & 1.6 & 27.5 \\
\hline \multicolumn{5}{|l|}{ PAP-1 (kg) } \\
\hline Weight lifted (kg) & $54.3 \pm 5.9^{b}$ & $39.4 \pm 12.5$ & 2.7 & 2.7 \\
\hline Repetitions & $19.6 \pm 0.8$ & $19.3 \pm 3.6$ & 0.1 & 0.1 \\
\hline
\end{tabular}

PAP-0.5: warm-up with PAP at moderate external speed $(0.5 \mathrm{~m} / \mathrm{s})$.

PAP-1: warm-up with PAP at high external speed $(1 \mathrm{~m} / \mathrm{s})$. a: difference $=(\mathrm{NL}-\mathrm{RL}) \times 100 / \mathrm{NL}$.

$b$ : denotes significant difference compared to regional level $(p<0.01)$.

c: denotes significant difference compared to PAP-1 $(p<0.01)$. 
Table 3

Effects of different post-activation potentiation (PAP) warm-ups on the repeated sprint ability test (RSA) in soccer players.

\begin{tabular}{|c|c|c|c|c|c|c|c|}
\hline & \multirow[t]{2}{*}{ CONTROL } & \multirow[t]{2}{*}{ PAP-1 } & \multirow[t]{2}{*}{ PAP-0.5 } & \multicolumn{2}{|c|}{ CONTROL versus PAP-1 } & \multicolumn{2}{|c|}{$\begin{array}{c}\text { CONTROL versus PAP- } \\
0.5\end{array}$} \\
\hline & & & & $\begin{array}{c}\text { Effect } \\
\text { size }\end{array}$ & $\begin{array}{c}\text { Difference }^{a} \\
(\%)\end{array}$ & $\begin{array}{c}\text { Effect } \\
\text { size }\end{array}$ & $\begin{array}{c}\text { Difference }^{a} \\
(\%)\end{array}$ \\
\hline RSAb (s) & $3.19 \pm 0.11$ & $3.20 \pm 0.14$ & $3.14 \pm 0.13$ & 0.1 & 0.3 & -0.4 & -1.6 \\
\hline NL & $3.15 \pm 0.06$ & $3.13 \pm 0.13^{b}$ & $3.05 \pm 0.07 c$ & -0.2 & -0.6 & -1.5 & -3.2 \\
\hline RL & $3.24 \pm 0.12$ & $3.26 \pm 0.14$ & $3.22 \pm 0.11$ & 0.2 & 0.6 & -0.2 & -0.6 \\
\hline RSAm(s) & $3.29 \pm 0.13$ & $3.31 \pm 0.16$ & $3.23 \pm 0.14$ & 0.1 & 0.6 & -0.1 & -1.8 \\
\hline NL & $3.24 \pm 0.08$ & $3.21 \pm 0.12^{\mathrm{b}}$ & $3.13 \pm 0.09 c$ & 0.2 & -0.9 & -1.3 & -3.4 \\
\hline RL & $3.34 \pm 0.14$ & $3.38 \pm 0.15$ & $3.32 \pm 0.12$ & -0.3 & 1.2 & -0.2 & -0.6 \\
\hline
\end{tabular}

CONTROL: warm-up without PAP. NL and RL: national and regional level soccer players, respectively.

PAP-0.5: warm-up with PAP at moderate external speed $(0.5 \mathrm{~m} / \mathrm{s})$. PAP-1: warm-up with

$P A P$ at high external speed $(1 \mathrm{~m} / \mathrm{s}) . R S A b=$ fastest sprint .

RSAm= mean RSA test time from 6 sprints. ${ }^{:}:(C O N T R O L-P A P) \times 100 / C O N T R O L$

$b$ and $c$ : denotes significant difference compared to $R L$ ( $p<0.05$ and 0.01 , respectively).

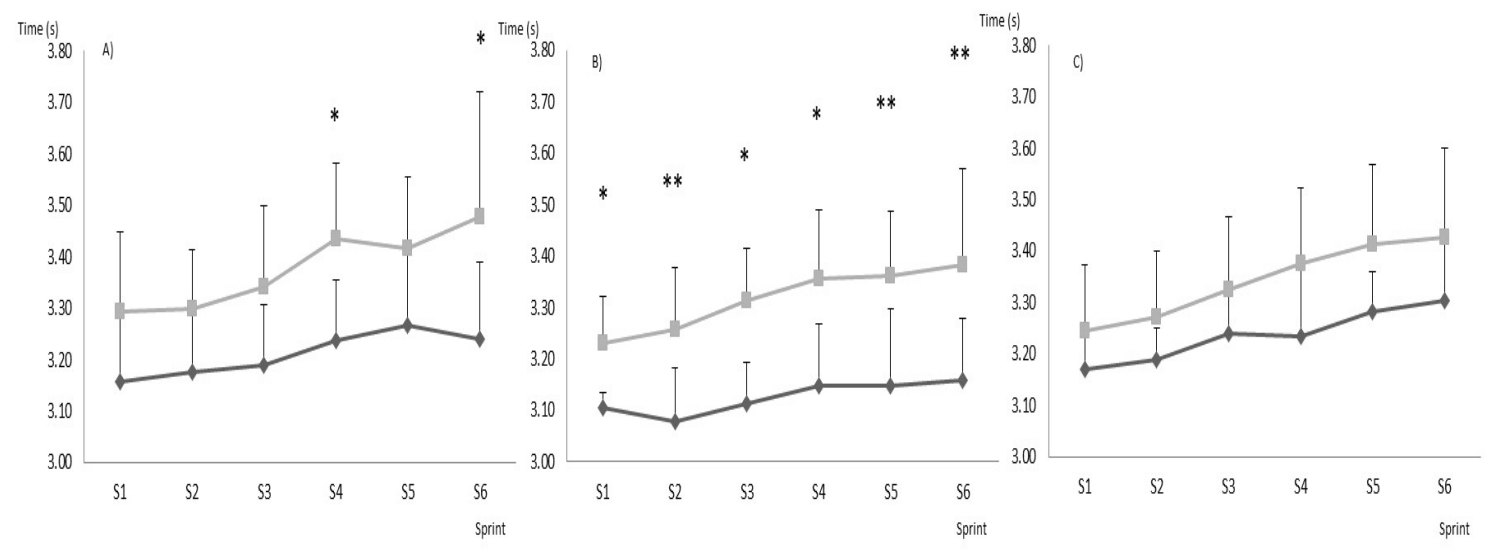

Figure 1

Effects of CONTROL (A), PAP-0.5 (B) and PAP-1 (C) on RSA test bouts (S1 to S6) in national ( ) and regional $(\square)$ level soccer players. CONTROL: warm-up without PAP;

PAP-0.5: warm-up with PAP at moderate external speed $(0.5 \mathrm{~m} / \mathrm{s})$; PAP-1: warm-up with

$P A P$ at high external speed $(1 \mathrm{~m} / \mathrm{s})$.

* and **: denotes significant differences between groups ( $p<0.05$ and 0.01, respectively). 


\section{Discussion}

The aim of the study was to compare the acute effects of a traditional warm-up with two PAP warm-up strategies on the RSA of soccer players from national and regional competitive levels. The main results showed that neither PAP1.0 nor PAP-0.5 resulted in substantial improvements in RSA performance when data of both NL and RL athletes were combined. However, the assessment of the two groups separately revealed meaningful improvements in both RSAb and RSAm for the NL athletes following the PAP-0.5 protocol.

Both PAP warm-up strategies failed to induce significant improvements in RSA compared to CONTROL (Table 3), contrasting with previous findings (Okuno et al., 2013; Zois et al., 2015). It is possible that the loads used in PAP1 ( $60 \%$ of $1 \mathrm{RM})$ and PAP-0.5 ( 90\% of $1 \mathrm{RM}) \mathrm{did}$ not allow optimal neuromuscular conditioning (Tillin and Bishop, 2009), thus limiting the ability of the neuromuscular system to achieve a higher physiological functional state (Hodgson et al., 2005). Alternatively, the recovery period between the end of the PAP warm-up and the RSA test did not allow optimal dissipation of fatigue (Behm et al., 2004). Future studies should consider these factors for the design of optimal PAP warm-up strategies for soccer players, without disregarding the individual responsiveness.

Although no differences were observed between NL and RL players in RSA performance in the CONTROL, the NL showed greater performance in RSAb and RSAm after PAP-0.5 (p $<0.01$ ) and PAP-1 ( $<$ 0.05) (Table 3). Moreover, after PAP-0.5, greater performance for all sprints was observed in NL compared to RL players (Figure 1, B). These results are similar to previous findings (Chiu et al., 2003; Wilson et al., 2013). Different factors may help to explain the higher PAP warm-up effects on NL compared to RL athletes, although the competition level itself may be a key factor (Robbins, 2005). Given the importance of RSA performance for soccer (Carling et al., 2012), the competitive level of athletes should be taken into account for the design of effective PAP warm-up strategies (Okuno et al., 2013; Zois et al., 2015) to maximize neuromuscular facilitation and minimize factors related with fatigue (Zois et al., 2011).

The higher strength level of NL compared to RL players (Table 2) may also contribute to the meaningfully greater effect of the PAP warm-up on RSA performance in the NL players (Gourgoulis et al., 2003). A greater strength level may be associated with a greater proportion of fast twitch muscle fibers, thus greater sensitivity to PAP effects (Hamada et al., 2000). Also, NL may have exhibited a greater recovery rate after the PAP warm-up and the potential neuromuscular and energy-related fatigue effects after PAP warm-ups (Bishop, 2003) might be lower in well-trained athletes (Chiu et al., 2003). Therefore, NL soccer players may also have exhibited a reduced fatigue effect after PAP warm-ups.

Moreover, the greater load used during PAP-0.5 compared to PAP-1 (Table 2) may also have contributed to a greater and meaningful improvement in RSA performance in NL compared to RL athletes, as in the former the peak of force and power may be attained with greater loads (Duthie et al., 2002). It is important to note that these neuromuscular variables are highly correlated with sprinting (Slawinski et al., 2010). However, the RSA performance depends on several factors (Bishop et al., 2011), not only maximal running sprint speed (Newman et al., 2004). Therefore, warm-up strategies that aside from neuromuscular potentiation may also delay neuromuscular fatigue (sprint decrement) and potentiate the metabolic machinery during RSA actions (Okuno et al., 2013), should be scientifically addressed in future studies. In addition, the effects of training RSA under potentiated condition should be addressed in future studies, as previous authors have reported limited effectiveness of RSA training per se in team sport athletes (Haugen et al., 2014; SoaresCaldeira et al., 2014).

A potential limitation of the present study deals with its ecological validity. To induce PAP effects soccer players completed explosive squats at maximal-voluntary velocity. Aside from logistical difficulties to implement them prior to games, safety considerations should be addressed by qualified personnel, which may be difficult especially in amateur clubs where a low strength and conditioning coach:player ratio exists. Thus, future studies should aim to establish ecologically valid PAP strategies, best suited to the warm-up conditions encountered before soccer competitive 
matches, such as plyometric drills or similar body-weight drills, emphasizing soccer-specific actions. Furthermore, a recent study (Gołaś et al., 2016) highlighted the need of individualizing the recovery time between conditioning exercise and targeted explosive motor performance, because athletes can differ in strength level, training experience and muscle fiber structure. Hence, this factor needs to be addressed in future studies in order to set optimal recovery times for players competing at different levels.

In conclusion, neither PAP-1.0 nor PAP0.5 resulted in substantial improvements in RSA performance when the data of both NL and RL athletes were combined. However, the assessment of the two groups separately revealed meaningful improvements in both RSAb and RSAm for the NL athletes following the PAP- 0.5 protocol. Therefore, adding a heavy strength-based conditioning exercise during the warm-up may induce a small improvement in RSA in soccer players, with up to large improvements in those from a greater competitive level.

\section{Acknowledgements}

All authors listed, made substantial, direct and intellectual contribution to the work, and approved it for publication. The authors wish to thank all the volunteers who participated in this study. The authors declare that they have no conflict of interest. There was no financial assistance with this project. The research material is original and not submitted elsewhere.

\section{References}

Aziz AR, Mukherjee S, Chia MY, Teh KC. Relationship between measured maximal oxygen uptake and aerobic endurance performance with running repeated sprint ability in young elite soccer players. $J$ Sports Med Phys Fitness, 2007; 47: 401-407

Baudry S, Duchateau J. Postactivation potentiation in a human muscle: effect on the rate of torque development of tetanic and voluntary isometric contractions. J Appl Physiol (1985), 2007; 102: 1394-1401

Behm DG, Button DC, Barbour G, Butt JC, Young WB. Conflicting effects of fatigue and potentiation on voluntary force. J Strength Cond Res, 2004; 18: 365-372

Bishop D. Warm up II: performance changes following active warm up and how to structure the warm up. Sports Med, 2003; 33: 483-498

Bishop D, Girard O, Mendez-Villanueva A. Repeated-sprint ability - part II: recommendations for training. Sports Med, 2011; 41: 741-756

Carling C, Le Gall F, Dupont G. Analysis of repeated high-intensity running performance in professional soccer. J Sports Sci, 2012; 30: 325-336

Chaouachi A, Castagna C, Chtara M, Brughelli M, Turki O, Galy O, Chamari K, Behm DG. Effect of warmups involving static or dynamic stretching on agility, sprinting, and jumping performance in trained individuals. J Strength Cond Res, 2010a; 24: 2001-2011

Chaouachi A, Manzi V, Wong Del P, Chaalali A, Laurencelle L, Chamari K, Castagna C. Intermittent endurance and repeated sprint ability in soccer players. J Strength Cond Res, 2010b; 24: 2663-2669

Chatzopoulos DE, Michailidis CJ, Giannakos AK, Alexiou KC, Patikas DA, Antonopoulos CB, Kotzamanidis CM. Postactivation potentiation effects after heavy resistance exercise on running speed. J Strength Cond Res, 2007; 21: 1278-1281

Chiu LZ, Fry AC, Weiss LW, Schilling BK, Brown LE, Smith SL. Postactivation potentiation response in athletic and recreationally trained individuals. J Strength Cond Res, 2003; 17: 671-677

Cronin JB, Mcnair PJ, Marshall RN. Is velocity-specific strength training important in improving functional performance? J Sports Med Phys Fitness, 2002; 42: 267-273

Duthie GM, Young WB, Aitken DA. The acute effects of heavy loads on jump squat performance: an evaluation of the complex and contrast methods of power development. J Strength Cond Res, 2002; 16: $530-538$

Faigenbaum AD, Bellucci M, Bernieri A, Bakker B, Hoorens K. Acute effects of different warm-up protocols on fitness performance in children. J Strength Cond Res, 2005; 19: 376-381

Gołaś A, Maszczyk A, Zajac A, Mikołajec K, Stastny P. Optimizing post activation potentiation for explosive 
activities in competitive sports. J Hum Kinet, 2016; 52: 96-106

Gonzalez-Badillo JJ, Pareja-Blanco F, Rodriguez-Rosell D, Abad-Herencia JL, Del Ojo-Lopez JJ, SanchezMedina L. Effects of velocity-based resistance training on young soccer players of different ages. J Strength Cond Res, 2015; 29: 1329-1338

Gourgoulis V, Aggeloussis N, Kasimatis P, Mavromatis G, Garas A. Effect of a submaximal half-squats warm-up program on vertical jumping ability. J Strength Cond Res, 2003; 17: 342-344

Gouvea AL, Fernandes IA, Cesar EP, Silva WA, Gomes PS. The effects of rest intervals on jumping performance: a meta-analysis on post-activation potentiation studies. J Sports Sci, 2013; 31: 459-467

Hamada T, Sale DG, Macdougall JD, Tarnopolsky MA. Postactivation potentiation, fiber type, and twitch contraction time in human knee extensor muscles. J Appl Physiol (1985), 2000; 88: 2131-2137

Haugen T, Tonnessen E, Leirstein S, Hem E, Seiler S. Not quite so fast: effect of training at $90 \%$ sprint speed on maximal and repeated-sprint ability in soccer players. J Sports Sci, 2014; 32: 1979-1986

Hodgson M, Docherty D, Robbins D. Post-activation potentiation: underlying physiology and implications for motor performance. Sports Med, 2005; 35: 585-595

Hoff J, Helgerud J. Endurance and strength training for soccer players: physiological considerations. Sports Med, 2004; 34: 165-180

Hopkins WG, Marshall SW, Batterham AM, Hanin J. Progressive statistics for studies in sports medicine and exercise science. Med Sci Sports Exerc, 2009; 41: 3-13

Kilduff LP, Owen N, Bevan H, Bennett M, Kingsley MI, Cunningham D. Influence of recovery time on postactivation potentiation in professional rugby players. J Sports Sci, 2008; 26: 795-802

Loturco I, Pereira LA, Cal Abad CC, Gil S, Kitamura K, Kobal R, Nakamura FY. Using Bar Velocity to Predict the Maximum Dynamic Strength in the Half-Squat Exercise. Int J Sports Physiol Perform, 2016; 11: 697700

Masamoto N, Larson R, Gates T, Faigenbaum A. Acute effects of plyometric exercise on maximum squat performance in male athletes. J Strength Cond Res, 2003; 17: 68-71

Newman MA, Tarpenning KM, Marino FE. Relationships between isokinetic knee strength, single-sprint performance, and repeated-sprint ability in football players. J Strength Cond Res, 2004; 18: 867-872

Okuno NM, Tricoli V, Silva SB, Bertuzzi R, Moreira A, Kiss MA. Postactivation potentiation on repeatedsprint ability in elite handball players. J Strength Cond Res, 2013; 27: 662-668

Pareja-Blanco F, Rodriguez-Rosell D, Sanchez-Medina L, Gorostiaga EM, Gonzalez-Badillo JJ. Effect of movement velocity during resistance training on neuromuscular performance. Int J Sports Med, 2014; 35: 916-924

Rampinini E, Bishop D, Marcora SM, Ferrari Bravo D, Sassi R, Impellizzeri FM. Validity of simple field tests as indicators of match-related physical performance in top-level professional soccer players. Int $J$ Sports Med, 2007; 28: 228-235

Rampinini E, Sassi A, Morelli A, Mazzoni S, Fanchini M, Coutts AJ. Repeated-sprint ability in professional and amateur soccer players. Appl Physiol Nutr Metab, 2009; 34: 1048-1054

Robbins DW. Postactivation potentiation and its practical applicability: a brief review. J Strength Cond Res, 2005; 19: 453-458

Sale D. Postactivation potentiation: role in performance. Br J Sports Med, 2004; 38: 386-387

Sanchez-Medina L, Gonzalez-Badillo JJ. Velocity loss as an indicator of neuromuscular fatigue during resistance training. Med Sci Sports Exerc, 2011; 43: 1725-1734

Sanchez-Medina L, Perez CE, Gonzalez-Badillo JJ. Importance of the propulsive phase in strength assessment. Int J Sports Med, 2010; 31: 123-129

Schilling BK, Stone MH. Stretching: Acute Effects on Strength and Power Performance. Strength $\mathcal{E}$ Conditioning Journal, 2000; 22: 44

Slawinski J, Bonnefoy A, Leveque JM, Ontanon G, Riquet A, Dumas R, Cheze L. Kinematic and kinetic comparisons of elite and well-trained sprinters during sprint start. J Strength Cond Res, 2010; 24: 896905

Soares-Caldeira LF, De Souza EA, De Freitas VH, De Moraes SM, Leicht AS, Nakamura FY. Effects of additional repeated sprint training during preseason on performance, heart rate variability, and stress 
symptoms in futsal players: a randomized controlled trial. J Strength Cond Res, 2014; 28: 2815-2826

Spencer M, Bishop D, Dawson B, Goodman C. Physiological and metabolic responses of repeated-sprint activities:specific to field-based team sports. Sports Med, 2005; 35: 1025-1044

Stolen T, Chamari K, Castagna C, Wisloff U. Physiology of soccer: an update. Sports Med, 2005; 35: 501-536

Taylor JM, Weston M, Portas MD. The effect of a short practical warm-up protocol on repeated sprint performance. J Strength Cond Res, 2013; 27: 2034-2038

Tillin NA, Bishop D. Factors modulating post-activation potentiation and its effect on performance of subsequent explosive activities. Sports Med, 2009; 39: 147-166

Turner AP, Bellhouse S, Kilduff LP, Russell M. Postactivation potentiation of sprint acceleration performance using plyometric exercise. J Strength Cond Res, 2015; 29: 343-350

Wilson JM, Duncan NM, Marin PJ, Brown LE, Loenneke JP, Wilson SM, Jo E, Lowery RP, Ugrinowitsch C. Meta-analysis of postactivation potentiation and power: effects of conditioning activity, volume, gender, rest periods, and training status. J Strength Cond Res, 2013; 27: 854-859

Woods K, Bishop P, Jones E. Warm-up and stretching in the prevention of muscular injury. Sports Med, 2007; 37: 1089-1099

Zois J, Bishop D, Aughey R. High-intensity warm-ups: effects during subsequent intermittent exercise. Int J Sports Physiol Perform, 2015; 10: 498-503

Zois J, Bishop DJ, Ball K, Aughey RJ. High-intensity warm-ups elicit superior performance to a current soccer warm-up routine. J Sci Med Sport, 2011; 14: 522-528

\section{Corresponding author:}

\section{Fábio Y. Nakamura}

Department of Medicine and Aging Sciences,

"G. d'Annunzio" University of Chieti-Pescara,

Via dei Vestini, 31, 66100 Chieti, Italy

E-mail: fabioy_nakamura@yahoo.com.br 